\title{
New horizons in the bacteriology, antimicrobial susceptibility and therapy of animal bite wounds
}

In the UK approximately 200000 persons suffer dog bites each year [1]; in the USA 4.7 million persons, or $1.8 \%$ of the population, are bitten annually and 800000 of these people seek medical attention [2,3]. The $20 \%$ of patients who seek medical attention are often those that are more seriously affected, have some complication such as infection and are the focus of published reports. In addition, 400000 persons in the USA annually are bitten by cats, 45000 by snakes and an unknown number by other domestic small pet mammals, feral and wild animals, and other human beings, the most aggressive animal of all. Most bite victims do not need, nor do they seek, medical attention. Relatively few large-scale, systematic studies address any of the various issues concerning the full spectrum of bacteriology of these wounds or the various elements of therapy $[4,5]$. Most data come from small studies or anecdotal case reports.

Dog bites occur more frequently in males $<20$ years old, whereas cat bites are more common in $30-40$ year-old women. More bites occur in the warm weather months and during middle afternoon around $4 \mathrm{pm}$. Most animal bite wounds are provoked and the biting animal is usually owned by, or known to, the victim. Bite wounds may be lacerations, evulsions, or punctures and may be accompanied by extensive trauma and crush injury. Infectious complications occur in $15-20 \%$ of injuries and include cellulitis, abscess, septic arthritis, osteomyelitis, and bacteraemia. From 1979 to 1994 , dog attacks resulted in 279 deaths in the USA [6], one-third of which were associated with pit bull terriers or rottweilers. Children account for $c .75 \%$ of fatalities and may also suffer more severe bite injuries, such as skull fractures, dural tears and blood vessel perforation $[6,7]$.

The bacteriology of paediatric and adult animal bite wounds has been the focus of several studies $[4,5]$ that noted aerobes in $85 \%$ of wounds and anaerobes in c. $50-65 \%$. Older studies classified isolates according to the available schemata, but new molecular methods have resulted in taxonomic changes that have revolutionised aerobic and anaerobic bacteriology, adding many new genera and species that were not previously recognised. Animal bite wounds in man are problematic, as the isolates are veterinary species that may be difficult to identify by colonial morphology and biochemical tests; they may not match descrip- tions of species usually causing human infection (some may be new species) and are often quite slow growing. Consequently, many laboratories may recognise and isolate only the predominant and familiar strains and miss some of the new and emerging pathogens.

In 1978, a study of 26 dog bite wounds noted that 23 $(88 \%)$ grew aerobes and $10(38 \%)$ grew anaerobes [5]. Brook [4] reported a series of 21 children bitten by animals (17 dog and four cat bites) with an average of one anaerobe and 1.8 aerobe isolates/specimen. He found Staphylococcus aureus, Pasteurella multocida and anaerobic cocci to be the most common isolates, together with four 'Bacteroides' species (now Prevotella melaninogenica, $P r$. intermedia and an unnamed species) and five fusobacteria. A research group from 18 Emergency Departments in academic centres in the USA has been formed to study infected dog and cat bite wounds in man, including the aerobic and anaerobic bacteriology of these wounds. A preliminary report on the first 50 cases in this study [8] found that $92 \%$ of wounds grew a total of 232 isolates - 155 aerobes and 77 anaerobes. Aerobes were isolated from all culture-positive wounds and anaerobes from $46 \%$ of dog bites (1-9 isolates/ wound) and $62 \%$ of cat bites (1-6 isolates/wound). The bacteriology of these wounds generally reflected the oral flora of the biting animal.

Among aerobes, Pasteurella spp. are recognised as common isolates from feline and canine bite wounds, but most studies have not differentiated them into the newly described species and $P$. multocida subspecies. Mutters et al. [9] recognised 13 taxa in DNA hybridisation studies. Subsequently, Holst et al. [10], in describing 159 human clinical isolates, noted various ecological differences and tropisms between certain species. P. multocida subsp. multocida and subsp. septica were often isolated from more serious infections, with the latter having a tropism for the central nervous system. Furthermore, $P$. canis was isolated only from dog bites while the other species were isolated routinely from cat bite wounds and scratches.

In early studies of anaerobes isolated from bite wounds, 'Bacteroides' (including many species since renamed) were the most common isolates, followed by fusobac- 
teria and peptostreptococci. These isolates did not produce $\beta$-lactamases. In our recent study [8] we identified the presence of many new genera and species. In addition, we have previously isolated 40 strains of Porphyromonas: 26 from 54 cat bites and 14 strains from $48 \mathrm{dog}$ bites. When further characterised by biochemical tests and arbitrarily primed PCR [11], Por. salivosa (11 isolates), Por. gingivalis (10) and Por. canoris (8) were most frequent; other isolates included Por. cangingivalis (3), Por. circumdentaria (2), Por. cansulci (1), Por. levii-like strains (1) and some unidentified species (4). Supplemented brucella agar supports growth of all these species, but $66 \%$ failed to grow on unsupplemented brucella blood agar, $38 \%$ on Columbia blood agar and $30 \%$ on tryptic soy blood agar without haemin or vitamin $\mathrm{K} 1$ supplementation [12]. In a comparison of the Rapid ID ANA II system, An-Ident panels and API ZYM strips, it was found that the latter performed best because of the presence of tests for trypsin and chymotrypsin; however, detection of glycosidase activity by the API ZYM strips was less sensitive than in the other systems. None of the three test systems was able to identify all the isolates or species [13]. The WEE-TAB system, a single tube, triple substrate system to detect certain pre-formed enzymes $(\mathrm{N}$-acetyl- $\beta$-D-glucosaminidase, $\alpha$-D-galactosidase, $\beta$-D-galactosidase, $\alpha$-fucosidase, trypsin-like activity and chymotrypsin) was recently evaluated and compared with the API ZYM and RapID-ANA II systems and was found to be useful and accurate in identification of most of these Porphyromonas spp. Por. circumdentaria and Por. cansulci could not be identified by any of the three systems because they are enzymically inert [12].

Several saccharolytic Bacteroides and Prevotella spp. are often isolated from $\operatorname{dog}$ and cat bite wounds. $B$. tectum is a frequent isolate and may be mistaken for Pr. bivia by colonial morphology and by test kit identification numbers; it may be differentiated by its growth in bile $20 \%$ and by aesculin hydrolysis [13]. PCR fingerprinting suggests as many as four subgroups in this heterogeneous species. Pr. heparinolytica, also a common animal bite wound isolate, may be confused with $B$. uniformis because of a positive indole test, but is sensitive to bile and easily distinguished from Bacteroides sensu stricto (the $B$. fragilis group). Pr. zoogleoformans is an occasional bite wound isolate that may also resemble $P r$. heparinolytica except for its negative indole test. $P r$. buccae and Pr. oris, which are also biochemically similar to $\mathrm{Pr}$. zoogleoformans and are indole negative, may be distinguished as they do not form a zoogleal mass in PRAS (pre-reduced anaerobically sterilised) broth media; also $\operatorname{Pr}$. buccae does not produce $\alpha$-Lfucosidase and Pr. oris isolates are positive for $\alpha-\mathrm{L}-$ arabinosidase [13]. Because of the slow growth of some of these bite isolates, microbiologists are advised to keep the plates for 7 days to ensure their isolation and accurate culture results.
The principles of therapy consist of irrigation, cautious debridement, elevation, recognition of and monitoring for common complications and appropriate antimicrobial therapy [14]. In-vitro studies with several newer antimicrobial agents have been published [15-18], but few clinical trials involving bite wound infections have been reported.

The new fluoroquinolones, levofloxacin, trovafloxacin, DU-6859a and Bay 12-8039, have excellent activity against almost all gram-positive and gram-negative aerobic isolates including all species and subspecies of Pasteurella, Actinobacillus and Haemophilus, Weeksella zoohelcum, EF-4a, Capnocytophaga spp., Eikenella corrodens, Neisseria weaveri, $S$. aureus, $S$. epidermidis and streptococci [15-17]. The older fluoroquinolones, ciprofloxacin and ofloxacin, are less active against streptococci. All fluoroquinolones have limited or poor in-vitro activity against $F$. nucleatum and other Fusobacterium spp., but good activity against $B$. tectum, Por. salivosa and Por. gingivalis. Trovafloxacin, Bay 12-8039 and DU 6859a exhibit good activity against peptostreptococci and other Porphyromonas spp. (levofloxacin, ofloxacin and ciprofloxacin are relatively less active), Pr. heparinolytica and other Prevotella spp. (ofloxacin and ciprofloxacin are less active).

Azithromycin is more active than erythromycin against many aerobes including $P$. multocida and $E$. corrodens. It is also two-four-fold more active than clarithromycin against aerobes such as Actinobacillus spp., Haemophilus spp., Moraxella spp. and Pasteurella spp., including all $P$. multocida subspp. For some isolates of $P$. canis, $P$ dagmatis, $P$ stomatis and $P$. testudinis, MICs of clarithromycin are $4-8 \mathrm{mg} / \mathrm{L}$. Clarithromycin is more active than azithromycin against some anaerobes such as B. tectum, Porphyromonas and Prevotella spp. [16]. It is unclear whether this difference in anaerobic activity is related to the presence of $\mathrm{CO}_{2}$ in the atmosphere of incubation, which lowers the agar surface $\mathrm{pH}$ and would have a greater effect on the activity of the dibasic azithromycin.

HMR 3004 is a new ketolide with a 3-keto group on a 14-membered erythronolide ring. It has greater activity against bite wound pathogens than its macrolide relatives. Almost all fastidious and aerobic isolates, including $P$. multocida, B. tectum, Prevotella spp., Porphyromonas spp. and peptostreptococci are inhibited at a concentration of $0.5 \mathrm{mg} / \mathrm{L}$, but $F$ nucleatum and other fusobacteria are more resistant $\left(\mathrm{MIC}_{90}\right.$ $16 \mathrm{mg} / \mathrm{L})$.

Cefuroxime and cefpodoxime have modest activity against gram-positive aerobes and limited activity against Prevotella spp. (other than Pr. heparinolytica), fusobacteria and some $B$. tectum strains [16]. Cefprozil has modest to poor activity against gram-positive 
aerobes and, like loracarbef, poor activity against peptostreptococci [18].

The choice of antimicrobial therapy favours an agent that is active against all the Pasteurella spp., S. aureus, streptococci and the anaerobic species such as $B$. tectum, Pr. heparinolytica, Por. salivosa, fusobacteria and peptostreptococci. Other considerations include the patient's allergy history as well as possible side-effects, drug interactions and other contra-indications.

ELLIE J. C. GOLDSTEIN

R.M. Alden Research Laboratory, Santa Monica-UCLA Medical Center, Santa Monica, CA 90404 and UCLA School of Medicine,

Los Angeles, CA 90074, USA e.mail EJCGHD@aol.com

I thank Diane M. Citron for her extensive co-operation and collaboration on all our laboratory studies and projects; Marie Hudspeth, Sharon Hunt Gerardo, C. Vreni Merriam and Katherine Nesbitt for technical assistance; and Judee Knight and Alice E. Goldstein for their support.

\section{References}

1. Moore F. "I've just been bitten by a dog." Surgical toilet appropriate antibiotics and advice to come back if infection develops. BMJ 1997; 314: 88-90.

2. Sosin DM, Sacks JJ, Sattin RW. Causes of nonfatal injuries in the United States, 1986. Acid Anal Prev 1992; 24: 685-687.

3. Sacks JJ, Kresnow M, Houston B. Dog bites: how big a problem? Injury Prev 1996; 2: 52-54.

4. Brook I. Microbiology of human and animal bite wounds in children. Pediatr Infect Dis $J$ 1987; 6: 29-32.

5. Goldstein EJC, Citron DM, Wield B et al. Bacteriology of human and animal bite wounds. $J$ Clin Microbiol 1978; 8: 667-672.

6. Lockwood R. Dog-bite-related fatalities-United States, $1995-$ 1996. MMWR 1997; 46: 463-467.

7. Brogan TV, Bratton SL, Dowd D, Hegenbarth MA. Severe dog bites in children. Pediatrics 1995; 96: 947-950.

8. Goldstein EJC, Citron DM, Nesbit $\mathrm{C}$ et al. Prevalence and characterization of anaerobic bacteria from 50 patients with infected cat and dog bite wounds. In: Eley $\mathrm{A}$, Bennett $\mathrm{K}$ (eds) Anaerobic pathogens. Sheffield, Sheffield Academic Press 1997: $177-185$

9. Mutters R, Ihm P, Pohl S, Frederiksen W, Mannheim W. Reclassification of the genus Pasteurella Trevisan 1887 on the basis of deoxyribonucleic acid homology, with proposals for the new species Pasteurella dagmatis, Pasteurella canis, Pasteurella stomatis, Pasteurella anatis, and Pasteurella langaa. Int J Syst Bacteriol 1985; 35: 309-322.

10. Holst E, Rollof J, Larrson L, Neilsen JP. Characterization and distribution of Pasteurella species recovered from infected humans. J Clin Microbiol 1992; 30: 2984-2987.

11. Citron DM, Hunt Gerardo S, Claros MC, Abrahamian F, Talan D, Goldstein EJC. Frequency of isolation of Porphyromonas species from infected dog and cat bite wounds in humans and their characterization by biochemical tests and arbitrarily primed-polymerase chain reaction fingerprinting. Clin Infect Dis 1996; 23 Suppl 1: S78-S82.

12. Hudspeth MK, Hunt Gerardo S, Citron DM, Goldstein EJC. Growth characteristics and a novel method for identification (the Wee-Tab system) of Porphyromonas species isolated from infected dog and cat bite wounds in humans. $J$ Clin Microbiol 1997; 35: 2450-2453.

13. Alexander CJ, Citron DM, Hunt Gerardo S, Claros MC, Talan D, Goldstein EJC. Characterization of saccharolytic Bacteroides and Prevotella isolates from infected dog and cat bite wounds in humans. J Clin Microbiol 1997; 35: 406-411.

14. Goldstein EJC. Bite wounds and infection. Clin Infect Dis 1992; 14: 633-640.

15. Goldstein EJC, Citron DM, Hunt Gerardo S, Hudspeth M Merriam CV. Comparative in vitro activities of DU-6859a levofloxacin, ofloxacin, sparfloxacin, and ciprofloxacin against 387 aerobic and anaerobic bite wound isolates. Antimicrob Agents Chemother 1997; 41: 1193-1195.

16. Goldstein EJC, Citron DM, Hudspeth M, Hunt Gerardo S Merriam CV. In vitro activity of Bay $12-8039$, a new 8methoxyquinolone, compared to the activities of 11 other oral antimicrobial agents against 390 aerobic and anaerobic bacteria isolated from human and animal bite wound skin and soft tissue infections in humans. Antimicrob Agents Chemother 1997; 41: 1552-1557.

17. Goldstein EJC, Citron DM, Hudspeth M, Hunt Gerardo S, Merriam CV. Trovafloxacin compared to levofloxacin, ofloxacin, ciprofloxacin, azithromycin and clarithromycin against unusual aerobic and anaerobic human and animal bite wound pathogens. $J$ Antimicrob Chemother (in press).

18. Goldstein EJC, Nesbit CA, Citron DM. Comparative in vitro activities of azithromycin, Bay y 3118 , levofloxacin, sparfloxacin and 11 other oral antimicrobial agents against 194 aerobic and anaerobic bite wound isolates. Antimicrob Agents Chemother 1995; 39: $1097-1100$. 